\title{
Effect of asymmetric concentration profile on thermal conductivity in Ge/SiGe superlattices
}

Konstanze R. Hahn, Stefano Cecchi, and Luciano Colombo

Citation: Appl. Phys. Lett. 108, 203102 (2016);

View online: https://doi.org/10.1063/1.4949491

View Table of Contents: http://aip.scitation.org/toc/apl/108/20

Published by the American Institute of Physics

\section{Articles you may be interested in}

Thermal transport in porous Si nanowires from approach-to-equilibrium molecular dynamics calculations Applied Physics Letters 109, 013107 (2016); 10.1063/1.4955038

Thermal conductivity of Si-Ge superlattices

Applied Physics Letters 70, 2957 (1998); 10.1063/1.118755

Thermal conductivity of Si/SiGe and SiGe/SiGe superlattices

Applied Physics Letters 80, 1737 (2002); 10.1063/1.1455693

Nanoscale thermal transport. II. 2003-2012

Applied Physics Reviews 1, 011305 (2014); 10.1063/1.4832615

Enhanced thermoelectric figure of merit in nanostructured $n$-type silicon germanium bulk alloy Applied Physics Letters 93, 193121 (2008); 10.1063/1.3027060

Nanoscale thermal transport

Journal of Applied Physics 93, 793 (2002); 10.1063/1.1524305

\section{Scilight}

Sharp, quick summaries illuminating the latest physics research

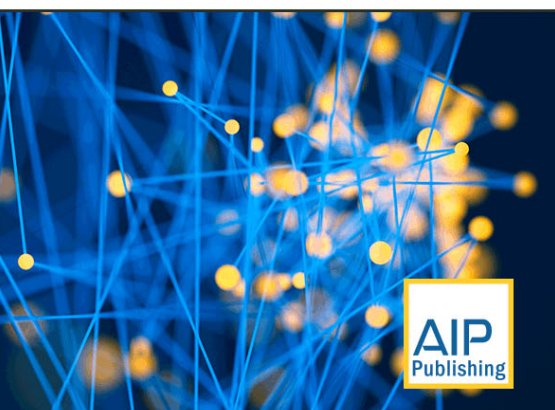




\title{
Effect of asymmetric concentration profile on thermal conductivity in $\mathrm{Ge} / \mathrm{SiGe}$ superlattices
}

\author{
Konstanze R. Hahn, ${ }^{1, a)}$ Stefano Cecchi, ${ }^{2}$ and Luciano Colombo ${ }^{1,3,4}$ \\ ${ }^{1}$ Department of Physics, University of Cagliari, Cittadella Universitaria, 09042 Monserrato (CA), Italy \\ ${ }^{2}$ Department of Epitaxy, Paul-Drude-Institut für Festkörperelektronik, Hausvogteiplatz 5-7, 10117 Berlin, \\ Germany \\ ${ }^{3}$ Institut de Cieǹcia de Materials de Barcelona (ICMAB-CSIC), Campus UAB, Bellaterra, 08193 Barcelona, \\ Spain \\ ${ }^{4}$ Catalan Institute of Nanoscience and Nanotechnology (ICN2), CSIC and The Barcelona Institute of Science \\ and Technology, Campus UAB, Bellaterra, 08193 Barcelona, Spain
}

(Received 8 February 2016; accepted 2 May 2016; published online 16 May 2016)

\begin{abstract}
The effect of the chemical composition in $\mathrm{Si} / \mathrm{Ge}$-based superlattices on their thermal conductivity has been investigated using molecular dynamics simulations. Simulation cells of $\mathrm{Ge} / \mathrm{SiGe}$ superlattices have been generated with different concentration profiles such that the $\mathrm{Si}$ concentration follows a step-like, a tooth-saw, a Gaussian, and a gamma-type function in direction of the heat flux. The steplike and tooth-saw profiles mimic ideally sharp interfaces, whereas Gaussian and gamma-type profiles are smooth functions imitating atomic diffusion at the interface as obtained experimentally. Symmetry effects have been investigated comparing the symmetric profiles of the step-like and the Gaussian function to the asymmetric profiles of the tooth-saw and the gamma-type function. At longer sample length and similar degree of interdiffusion, the thermal conductivity is found to be lower in asymmetric profiles. Furthermore, it is found that with smooth concentration profiles where atomic diffusion at the interface takes place the thermal conductivity is higher compared to systems with atomically sharp concentration profiles. Published by AIP Publishing.
\end{abstract}

[http://dx.doi.org/10.1063/1.4949491]

A still limiting factor for the large scale use of thermoelectric devices is their comparatively high cost/Watt ratio. It is thus of great interest to optimize the efficiency of the thermoelectric material by maximizing its figure of merit $Z T .^{1-3}$ A possible strategy to increase $Z T$ is the introduction of impurities, for example by alloying as indeed observed in $\mathrm{Si}_{1-x} \mathrm{Ge}_{x}$ alloys. $^{4-8}$

The reduction of thermal conductivity in alloys is a result of high-frequency phonon scattering at impurity atoms. Alloying however does not affect mid- and lowfrequency phonons. ${ }^{1,2}$ In order to suppress the propagation of such phonons, it is useful to generate nanofeatures as confirmed by several theoretical and experimental studies. ${ }^{6,8-12}$ The decrease of the thermal conductivity $\kappa$ results from increased phonon scattering at the interfaces introduced by nanograins, nanowires, or superperiodicity.

In the last decades, numerous studies have been dedicated to the investigation of Si/Ge superlattices. ${ }^{12-20}$ In particular, it has been shown that diffusion of the counter atoms in $\mathrm{Si} / \mathrm{Ge}$ superlattices can result in scattering of phonons at all frequencies. ${ }^{20,21}$ Recently, $\mathrm{Si} / \mathrm{SiGe}$ superlattices have been generated experimentally with a graded (tooth-saw) concentration profile providing a lower thermal conductivity with respect to the thermal conductivity in the homogeneous SiGe alloy. ${ }^{15}$ However, the effect and interplay of different phonon scattering processes in such superlattice structures is still not well understood.

In this study, we have used molecular dynamics simulations to estimate the thermal conductivity in $\mathrm{Ge} / \mathrm{SiGe}$

${ }^{a)}$ Electronic mail: konstanze.hahn@dsf.unica.it superlattices with different sharp as well as broad concentration profiles that imitate diffusion processes at the interface region. The choice of such a system is inspired by the work of Llin and coauthors, ${ }^{13}$ where multilayers of $\mathrm{Ge} / \mathrm{SiGe}$ have been generated experimentally for industrially applicable thermoelectric devices.

The Ge/SiGe superlattices are oriented with the crystallographic (001) plane orthogonal to the heat flux and are built of several layers of crystalline Ge and a barrier consisting of a SiGe alloy. To account for periodic boundary conditions, the in-plane lattice spacing has been equally set to $a_{\mathrm{Ge}, 0}$ $=5.6567 \AA$ for both $\mathrm{Ge}$ and SiGe sections. The out-of-plane lattice parameter has been calculated from the elastic properties of the material as described previously ${ }^{22}$ and has been adjusted for each corresponding Si concentration.

One unit block consisting of a $\mathrm{Ge}$ and a SiGe alloy section is replicated in $z$-direction (direction of the heat flux) to generate superlattices. The unit block consists of $n_{\mathrm{p}}=24$ biatomic layers corresponding to $6.7 \mathrm{~nm}$. The atomic configuration in the unit blocks has been generated according to four different concentration profiles which are based (i) on a step-like function (step), (ii) on a Gaussian distribution (gauss), (iii) on a gamma-type distribution (gamma), and (iv) on a tooth-saw profile (tooth). In all samples, the total average Ge concentration is maintained at $83.3 \%$, and the maximum concentration of $\mathrm{Si}$ in the $\mathrm{SiGe}$ alloy reaches $50 \%$ according to recent experimental studies. ${ }^{13}$ The unit blocks have been created by random substitution of $\mathrm{Ge}$ atoms with $\mathrm{Si}$, where the number of substitutions for each biatomic layer is defined by the corresponding concentration profile. 
The concentration profile of the step function (Fig. 1(a)) is defined by

$$
c_{\mathrm{Si}}= \begin{cases}0 & n_{i} \leq n_{\mathrm{Ge}} \\ 0.5 & n_{i}>n_{\mathrm{Ge}}\end{cases}
$$

where $n_{i}$ is the index of the biatomic layer and $n_{\mathrm{Ge}}$ is the number of biatomic layers corresponding to crystalline Ge; in this case, $n_{\mathrm{Ge}}=16$.

The gauss profile (Fig. 1(a)) is described by

$$
c_{\mathrm{Si}}=\frac{1}{2} \exp \left\{-\frac{1}{2} \frac{\left(n_{i}-\mu\right)^{2}}{\sigma^{2}}\right\},
$$

where $\mu$ is the center of the SiGe section, here $\mu=20$ (number of biatomic layers). In the performed calculations, $\sigma$ has been set to 3.2 (corresponding to $0.9 \mathrm{~nm}$ ).

The gamma profile (Fig. 1(b)) has been generated based on

\section{a) symmetric profile}
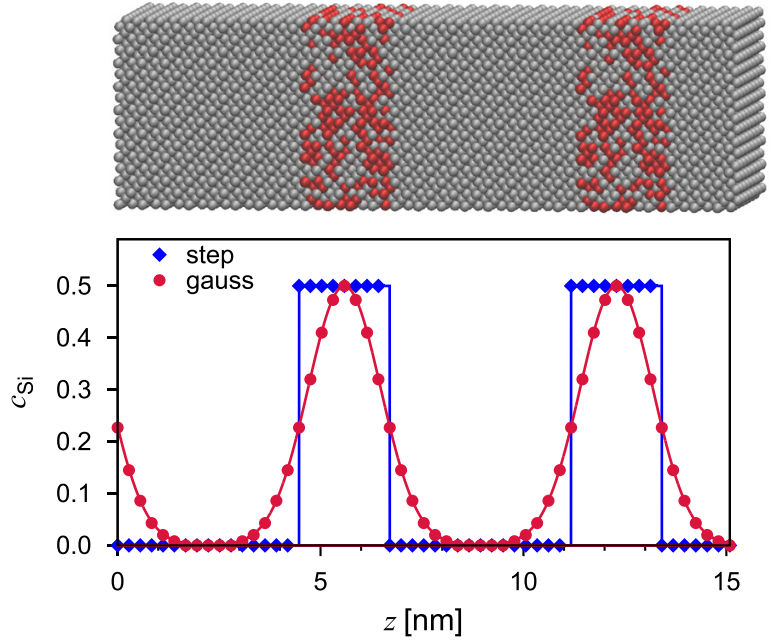

\section{b) asymmetric profile}

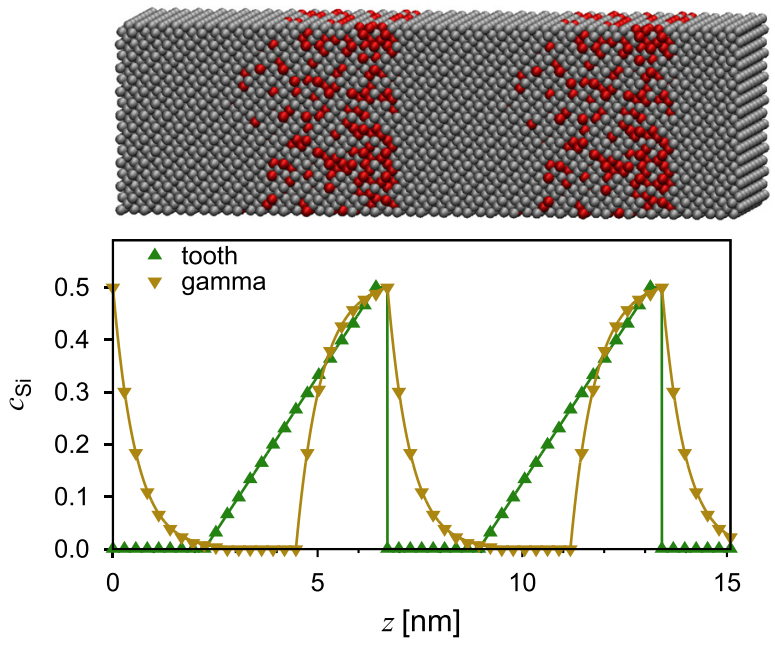

FIG. 1. Section of the simulation cells of the (a) step and the (b) tooth samples and the corresponding concentration profiles of (a) symmetric and (b) asymmetric samples.

$$
c_{\mathrm{Si}}= \begin{cases}\frac{1}{2} \mathrm{e}^{-\frac{1}{2} \cdot n_{i}} & n_{i} \leq n_{\mathrm{Ge}} \\ \frac{1}{2}\left(1-\mathrm{e}^{-\frac{\left(n_{i}-n_{\mathrm{Ge}}\right)}{\gamma}}+\frac{1}{n_{\mathrm{SiGe}}} \mathrm{e}^{\left.-\frac{n_{\mathrm{SiGe}}\left(n_{i}-n_{\mathrm{Ge})}\right.}{\gamma}\right)}\right. & n_{i}>n_{\mathrm{Ge}},\end{cases}
$$

with $n_{\mathrm{Ge}}=16$ and the number of nominal SiGe layers $n_{\mathrm{SiGe}}=n_{\mathrm{p}}-n_{\mathrm{Ge}}=8$. The parameter $\gamma$ has been set to 2.15 (corresponding to $0.6 \mathrm{~nm}$ ).

The tooth profile (Fig. 1(b)) has been generated according to

$$
c_{\mathrm{Si}}= \begin{cases}0 & n_{i} \leq n_{\mathrm{Ge}} \\ \frac{0.5}{\left(n_{\mathrm{p}}-n_{\mathrm{Ge}}\right)} \cdot\left(n_{i}-n_{\mathrm{Ge}}\right) & n_{i}>n_{\mathrm{Ge}} .\end{cases}
$$

In order to obtain a total Ge concentration of $83.3 \%$, the number of crystalline Ge layers is set to $n_{\mathrm{Ge}}=8$.

Molecular dynamics simulations have been performed using the LAMmPS code. ${ }^{23}$ Interatomic forces have been described applying the Tersoff pair potential ${ }^{24}$ which has been shown to represent reasonably well the mechanical and thermal properties of $\mathrm{Si}$ and Ge materials. ${ }^{8,21,22,25-28}$ The bond-order Tersoff potential is known to overestimate the frequencies of transverse acoustic modes. ${ }^{29}$ This can result in an underestimation of the thermal conductivity at low temperatures $(<400 \mathrm{~K})$. Nevertheless, it performs well in predicting lattice thermal conductivity of SiGe alloys in very good agreement with ab initio calculations. ${ }^{8}$

Approach-to-equilibrium molecular dynamics (AEMD) ${ }^{30}$ have been used to calculate the thermal conductivity following the same protocol already adopted for $\mathrm{SiGe}$ heterosystems. ${ }^{8,31}$

The thermal conductivity $\kappa$ has been estimated in samples with different cell lengths $L_{z}$ from 200 to $600 \mathrm{~nm}$. The error of the fitted value of $\kappa$ for all samples presented here is smaller than $0.001 \mathrm{~W} / \mathrm{mK}$. It is the standard error of the least squares fit of the simulation data to Fourier's transport equation as used in the AEMD method. ${ }^{30}$ In this range, the evolution of the inverse thermal conductivity $1 / \kappa$ with the inverse sample length $1 / L_{z}$ follows a linear trend (Fig. 2). The linear relationship between $1 / \kappa$ and $1 / L_{z}$ can be expressed according to $\frac{1}{\kappa}=\frac{1}{\kappa_{\infty}}\left(1+\frac{\lambda}{L_{z}}\right)$, where $\lambda$ is a characteristic length, and

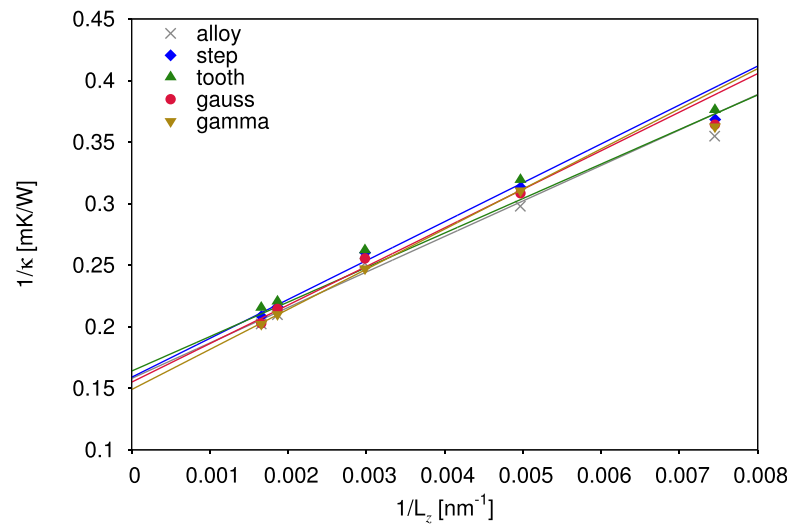

FIG. 2. Inverse thermal conductivity $1 / \kappa$ of $\mathrm{Ge} / \mathrm{SiGe}$ superlattices with different concentration profiles as a function of the inverse simulation cell length $1 / L_{z}$. 
it indicates that phonon properties can be estimated well by an average value. ${ }^{31,32}$ From the linear trend, the bulk thermal conductivity $\kappa_{\infty}$ can be approximated. It is estimated to be between 6.11 (tooth) and $6.70 \mathrm{~W} / \mathrm{mK}$ (gamma, Table I).

The thermal conductivity of the atomically sharp concentration profiles step $(6.29 \mathrm{~W} / \mathrm{mK})$ and tooth $(6.11 \mathrm{~W} / \mathrm{mK})$ lies below the value of the homogeneous alloy with the same average Ge concentration of $83.3 \%(6.34 \mathrm{~W} / \mathrm{mK})$. This indicates that in these samples scattering of phonons at the interface, contributing to the thermal boundary resistance in such samples, is more prominent than alloy scattering on impurity atoms in the homogeneous $\mathrm{Si}_{0.17} \mathrm{Ge}_{0.83}$ sample. On the other hand, when diffusion at the interface takes place, as it is the case for the smooth profiles gauss and gamma, the thermal conductivity increases to 6.43 and $6.70 \mathrm{~W} / \mathrm{mK}$, respectively.

The effect of the sample length on the phonon spectrum has been further analyzed by an accumulation function which represents the fraction of the thermal conductivity that is accumulated with increasing simulation cell length $L_{z} \cdot{ }^{31,32}$ The sample length in this case can be correlated to the mean free path of phonons. Thus, the accumulation function represents the contribution to the thermal conductivity of phonons that have a mean free path smaller than the corresponding sample length $L_{z}$. Assuming a normal distribution $f(\Lambda)$ of the mean free path according to $f(\Lambda)=\frac{1}{\sigma \sqrt{2 \pi}} \exp \left(-\frac{(\Lambda-\tilde{\mu})^{2}}{2 \sigma^{2}}\right)$, the accumulation function can be expressed by $\frac{\kappa(\Lambda)}{\kappa_{\infty}}=\frac{1}{2}\left[1+\operatorname{erf}\left(\frac{\Lambda-\tilde{\mu}}{\sigma \sqrt{2}}\right)\right]$, where $\Lambda$ is the logarithm of the dimensionless simulation cell length $\left(\Lambda=\log \left(\frac{L_{z}}{\lambda}\right)\right)$ and $\tilde{\mu}$ and $\sigma$ the mean and the standard deviation, respectively, of the normal distribution of the phonon mean free path. The statistical value $\tilde{\mu}$ can be translated into $\mu=\lambda \cdot 10^{\tilde{\mu}}$ which corresponds to the average value of the phonon mean free path of the system. ${ }^{31}$ Fig. 3 shows the accumulation function with the optimized parameters reported in Table I.

In all simulated $\mathrm{Ge} / \mathrm{SiGe}$ superlattices, the average phonon mean free path is found to be larger $(185-208 \mathrm{~nm})$ with respect to the SiGe alloy $(176 \mathrm{~nm}$, Table I). This suggests that randomly distributed atomic impurities, as found in the SiGe alloy, lead to a higher reduction of the phonon mean free path than defined interfaces with more pronounced atomic mismatch and is consistent with Garg et al. ${ }^{7}$ The highest average phonon mean free path is found in the gamma sample with $208 \mathrm{~nm}$ followed by the one of the gauss sample $(193 \mathrm{~nm})$. In both profiles, some respective counter

TABLE I. Bulk thermal conductivity for samples with $L_{z}$ between 200 and $600 \mathrm{~nm}$ with the average mean free path $\mu$, obtained from the accumulation function, and the $68 \%$ confidential interval (CI) assuming a normal distribution of the phonon mean free path. The goodness of fit of the accumulation function is described by $R^{2}$.

\begin{tabular}{lcclc}
\hline \hline Profile & $\kappa_{\infty}(\mathrm{W} / \mathrm{mK})$ & $\mu(\mathrm{nm})$ & $68 \% \mathrm{CI}(\mathrm{nm})$ & $R^{2}$ \\
\hline Alloy & $6.34 \pm 0.33$ & $176.4 \pm 0.02$ & $33 \div 945$ & 0.991 \\
Step & $6.29 \pm 0.29$ & $189.7 \pm 0.02$ & $34 \div 1045$ & 0.991 \\
Gauss & $6.43 \pm 0.31$ & $193.0 \pm 0.02$ & $35 \div 1055$ & 0.990 \\
Gamma & $6.70 \pm 0.06$ & $207.9 \pm 0.01$ & $37 \div 1171$ & 0.996 \\
Tooth & $6.11 \pm 0.16$ & $184.9 \pm 0.01$ & $33 \div 1019$ & 0.995 \\
\hline \hline
\end{tabular}

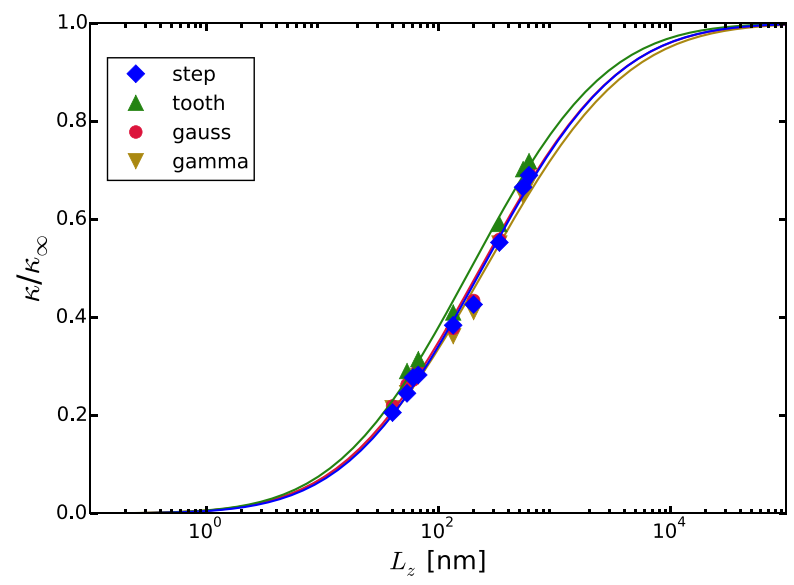

FIG. 3. Accumulation function of the thermal conductivity of $\mathrm{Ge} / \mathrm{SiGe}$ superlattices with different concentration profiles as a function of the simulation cell length $L_{z}$ (i.e., mean free path of involved phonons).

atoms diffuse into the other regime at the interface. This generates channels for the phonons to travel through the interfaces which are not atomically sharp as in the step and tooth profile. In addition, in the gamma sample, the region with a Si content $<10 \%$ is larger than in the gauss profile reducing the scattering at impurity atoms which results in a larger phonon mean free path. Assuming a normal distribution of the phonon mean free path, the $68 \%$ confidence interval (CI) is similar in all simulated concentration profiles (Table I).

The average Ge content (83.3\%) of the simulated superlattices is in the range where the thermal conductivity of the homogeneous alloy is already remarkably decreased compared to the pure $\mathrm{Si}$ and $\mathrm{Ge}^{4-8}$ Furthermore, the atomic mismatch between the $\mathrm{Ge}$ and the $\mathrm{Si}_{0.5} \mathrm{Ge}_{0.5}$ section is reduced with respect to the mismatch between pure $\mathrm{Si}$ and Ge. This results in a very small variation of bulk thermal conductivity with the different concentration profiles simulated here. Approximation of the latter from the linear relationship of $1 / \kappa$ to $1 / L_{z}$ is not very precise to accurately capture differences in the thermal transport properties of the different samples. The thermal conductivity has thus been compared directly between the different concentration profiles at three finite simulation cell lengths $L_{z}=335,537$, and $600 \mathrm{~nm}$ where the simulations have been run until the rate of time variation of the thermal conductivity resulted to be less than $0.06 \mathrm{~W} / \mathrm{mK}$ per ns. This typically was observed not before $\sim 4$ ns of simulation time for all three cell lengths. Since we are rather interested in relative differences than in absolute values, $\kappa$ has been normalized to the value of the SiGe alloy at the same cell size. The results are summarized in Fig. 4. The ordering along the horizontal axis follows the increasing asymmetric character of the $\mathrm{Si} / \mathrm{SiGe}$ interfaces (see Fig. 1).

Independently of the sample length, the thermal conductivity in samples with sharp concentration profiles step and tooth is lower compared to the smooth profiles gauss and gamma. At a sample length of $L_{z}=335$ and $537 \mathrm{~nm}$, it even reaches higher values than the homogeneous $\mathrm{SiGe}$ alloy with the same total Ge concentration of $83.3 \%$. The same is observed for the bulk thermal conductivity $\kappa_{\infty}$ (see Table I).

Lower conductivities in the samples with sharp profiles step and tooth result from enhanced phonon scattering at the interface due to atomic mismatch. Atomic mismatch is 


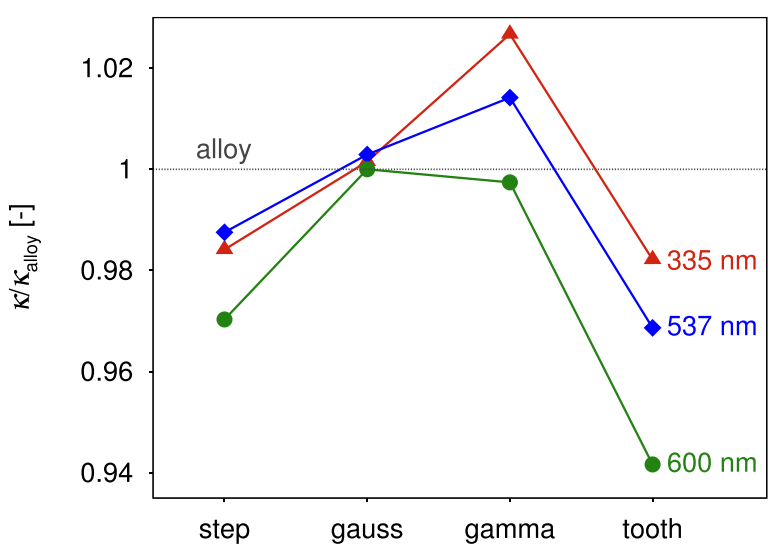

FIG. 4. Thermal conductivity of Ge/SiGe superlattices with different concentration profiles estimated at a simulation cell length $L_{z}=335,537$, and $600 \mathrm{~nm}$. Thermal conductivity in systems with sharp interfaces (step and tooth) is always lower than in systems where interdiffusion at the interface takes place (gauss and gamma).

reduced when interdiffusion at the interface is present resulting in an increased thermal conductivity in the samples with gauss and gamma profiles. The present simulation results suggest that in these concentration profiles the thermal boundary resistance is comparatively smaller and thermal transport is mainly affected by alloy scattering at impurity atoms which affects high-frequency phonons. ${ }^{15,16}$

At $L_{z}=335 \mathrm{~nm}$, similar values are found for the thermal conductivity in the step and tooth profile indicating that the asymmetry has only marginal effect on the thermal transport. This shows that the reduced thermal conductivity with respect to the homogeneous alloy mainly results from interface scattering directed by the atomic mismatch which is the same in the two profiles at the $\mathrm{Ge} / \mathrm{Si}_{0.5} \mathrm{Ge}_{0.5}$ interface.

Increasing the sample length, however, leads to a sizable reduction of the thermal conductivity in samples with asymmetric profiles tooth and gamma with respect to the alloy and the symmetric profiles step and tooth. The larger is the sample length, the higher is the number of interfaces (i.e., active scattering centers) that a phonon mode must pass through. This reflects in an overall decrease of the (normalized) thermal conductivity. Fig. 4 clearly suggests that asymmetric profiles affect thermal conduction to a considerably larger extent than symmetric ones. This is likely due to a reduction of the correspondent tunneling probability of incoming phonons. As a matter of fact, asymmetric profiles have the same height of their symmetric counterparts, while showing a larger width. This is especially true for the tooth profile which, in fact, corresponds to the comparatively smaller conductivity. These findings are in agreement with experimental evidence in samples where gradients in the concentration profiles resulted in a lower thermal conductivity compared to conventional symmetric Ge/SiGe superlattices. ${ }^{15}$ Previously, ab initio calculations have been used to proof experimental evidence that in $\mathrm{Si} / \mathrm{Ge}$ superlattices segregation of $\mathrm{Ge}$ in the $\mathrm{Si}$ layer leads to a decrease of the thermal conductivity with respect to an atomically sharp interface. ${ }^{20}$ This type of segregation results in an asymmetric concentration profile which can be compared to the tooth profile simulated here. The results shown here are thus in agreement with the latter study showing a lower thermal conductivity of the tooth profile with respect to the step profile at a sufficiently large sample length $\left(L_{z}>500 \mathrm{~nm}\right)$. A direct comparison of the two studies, however, is critical, since there the superlattice layers consisted of pure $\mathrm{Si}$ and $\mathrm{Ge}$, respectively, whereas here we simulated boundaries between Ge and the SiGe alloy.

In conclusion, we have shown that the interface diffusion leads to an increase of the thermal conductivity with respect to sharp $\mathrm{Ge} / \mathrm{SiGe}$ boundaries. At sufficiently long length $\left(L_{z}=600 \mathrm{~nm}\right)$, the thermal conductivity in systems with asymmetric concentration profiles is lower than for symmetric profiles with comparable interdiffusion at the interface. This effect is attributed to possible tunneling of phonons through the interface as a result of the symmetric composition around the interface. These findings give interesting insight into the effect of concentration profiles on the thermal conductivity in Ge/SiGe superlattices which are hard to be observed experimentally due to small variations in the absolute values. The small changes in heat transport properties predicted by our calculations suggest that for systems similar to the ones investigated here the actual concentration profile only plays a marginal role in affecting the thermal conductivity and, in turn, indicates that the level of accuracy in the present state-of-the-art fabrication of such superlattices is sufficient to generate materials with desired heat transport properties. Nevertheless, the results of this study are a basis for an extended exploration of the thermal transport properties of such $\mathrm{Si} / \mathrm{Ge}$-based superlattices as a function of their composition, interface properties, and device dimension.

${ }^{1}$ M. S. Dresselhaus, G. Chen, M. Y. Tang, R. G. Yang, H. Lee, D. Z. Wang, Z. F. Ren, J. P. Fleurial, and P. Gogna, Adv. Mater. 19, 1043 (2007).

${ }^{2}$ A. J. Minnich, M. S. Dresselhaus, Z. F. Ren, and G. Chen, Energy Environ. Sci. 2, 466 (2009).

${ }^{3}$ J. Karni, Nat. Mater. 10, 481 (2011).

${ }^{4}$ H. Stöhr and W. Klemm, Z. Anorg. Allg. Chem. 241, 305 (1939).

${ }^{5}$ B. Abeles, Phys. Rev. 131, 1906 (1963).

${ }^{6}$ X. W. Wang, H. Lee, Y. C. Lan, G. H. Zhu, G. Joshi, D. Z. Wang, J. Yang, A. J. Muto, M. Y. Tang, J. Klatsky, S. Song, M. S. Dresselhaus, G. Chen, and Z. F. Ren, Appl. Phys. Lett. 93, 193121 (2008).

${ }^{7}$ J. Garg, N. Bonini, B. Kozinsky, and N. Marzari, Phys. Rev. Lett. 106, 045901 (2011).

${ }^{8}$ C. Melis and L. Colombo, Phys. Rev. Lett. 112, 065901 (2014).

${ }^{9}$ S. K. Bux, R. G. Blair, P. K. Gogna, H. Lee, G. Chen, M. S. Dresselhaus, R. B. Kaner, and J.-P. Fleurial, Adv. Funct. Mater. 19, 2445 (2009).

${ }^{10}$ Y. P. He, D. Donadio, and G. Galli, Nano Lett. 11, 3608 (2011).

${ }^{11}$ S. Bathula, M. Jayasimhadri, N. Singh, A. K. Srivastava, J. Pulikkotil, A. Dhar, and R. C. Budhani, Appl. Phys. Lett. 101, 213902 (2012).

${ }^{12}$ I. Savić, D. Donadio, F. Gygi, and G. Galli, Appl. Phys. Lett. 102, 073113 (2013).

${ }^{13}$ L. F. Llin, A. Samarelli, S. Cecchi, T. Etzelstorfer, E. M. Gubler, D. Chrastina, G. Isella, J. Stangl, J. M. R. Weaver, P. S. Dobson, and D. J. Paul, Appl. Phys. Lett. 103, 143507 (2013).

${ }^{14}$ E. Landry and A. McGaughey, Phys. Rev. B 79, 075316 (2009).

${ }^{15}$ P. Ferrando-Villalba, A. F. Lopeandía, F. X. Alvarez, B. Paul, C. de Tomás, M. I. Alonso, M. Garriga, A. R. Goñi, J. Santiso, G. Garcia, and J. Rodriguez-Viejo, Nano Res. 8, 2833 (2015).

${ }^{16}$ P. Chen, T. Etzelstorfer, F. Hackl, N. A. Katcho, H.-T. Chang, L. Nausner, S.-W. Lee, T. Fromherz, J. Stangl, O. G. Schmidt, N. Mingo, and A. Rastelli, Phys. Status Solidi A 213, 533 (2016).

${ }^{17}$ S.-M. Lee, D. G. Cahill, and R. Venkatasubramanian, Appl. Phys. Lett. 70, 2957 (1997)

${ }^{18}$ S. T. Huxtable, A. R. Abramson, C.-L. Tien, A. Majumdar, C. LaBounty, X. Fan, G. Zeng, J. E. Bowers, A. Shakouri, and E. T. Croke, Appl. Phys. Lett. 80, 1737 (2002).

${ }^{19}$ G. Pernot, M. Stoffel, I. Savic, F. Pezzoli, P. Chen, G. Savelli, A. Jacquot, J. Schumann, U. Denker, I. Monch, C. Deneke, O. G. Schmidt, J. M. 
Rampnoux, S. Wang, M. Plissonnier, A. Rastelli, S. Dilhaire, and N. Mingo, Nat. Mater. 9, 491 (2010).

${ }^{20}$ P. Chen, N. A. Katcho, J. P. Feser, W. Li, M. Glaser, O. G. Schmidt, D. G. Cahill, N. Mingo, and A. Rastelli, Phys. Rev. Lett. 111, 115901 (2013).

${ }^{21}$ R. Dettori, C. Melis, and L. Colombo, Eur. Phys. J. B 88, 27 (2015).

${ }^{22}$ K. R. Hahn, M. Puligheddu, and L. Colombo, Phys. Rev. B 91, 195313 (2015).

${ }^{23}$ S. Plimpton, J. Comput. Phys. 117, 1 (1995).

${ }^{24}$ J. Tersoff, Phys. Rev. B 39, 5566 (1989).

${ }^{25}$ S. Cook and P. Clancy, Phys. Rev. B 47, 7686 (1993).
${ }^{26}$ L. J. Porter, S. Yip, M. Yamaguchi, H. Kaburaki, and M. Tang, J. Appl. Phys. 81, 96 (1997).

${ }^{27}$ X. Li and R. Yang, Phys. Rev. B 86, 054305 (2012).

${ }^{28}$ X. Li and R. Yang, J. Phys.: Condens. Matter 24, 155302 (2012).

${ }^{29}$ L. J. Porter, J. F. Justo, and S. Yip, J. Appl. Phys. 82, 5378 (1997).

${ }^{30}$ C. Melis, R. Dettori, S. Vandermeulen, and L. Colombo, Eur. Phys. J. B 87, 96 (2014).

${ }^{31}$ K. R. Hahn, C. Melis, and L. Colombo, Eur. Phys. J. B 87, 150 (2014).

${ }^{32}$ D. P. Sellan, E. S. Landry, J. E. Turney, A. J. H. McGaughey, and C. H. Amon, Phys. Rev. B 81, 214305 (2010). 